\title{
Proposta de Conversor de Potência Parcial e o Efeito de Distorção Harmônica
}

\author{
Galvão, T. M. ; Simonetti, D.S.L. \\ Programa de Pós-Graduação em Engenharia Elétrica, Universidade Federal do Espírito Santo, Vitória, ES, Brasil. \\ * e-mail: thiago.galvao@aluno.ufes.br
}

\begin{abstract}
Resumo
Neste trabalho foram propostas duas situações: i) a principal corresponde em validar através de simulação no software MATLAB/Simulink o uso de um simples conversor cuja potência nominal de ensaio é fracionada se comparada com a elevada potência de ensaio do conversor Back-to-Back (B2B), encontrado na literatura técnica em [1-3]. O conversor é parte fundamental no ensaio de carregamento do transformador sob teste (TUT - Transformer Under Test). Foi medida a potência aparente na saída do conversor, além das potências ativa e reativa provenientes da rede conectada com o sistema. O resultado do valor de potência aparente medido no conversor é reduzido se comparada com a potência do TUT, concluindo ser possível realizar o mesmo ensaio substituindo um conversor de $75 \mathrm{kVA}$ por um de $5 \mathrm{kVA}$. A outra proposição refere-se em: ii) analisar no aspecto da qualidade da energia o indicador de distorção harmônica de tensão, medida na saída do conversor de potência. Para esta proposta do trabalho, os testes foram realizados em três etapas, com alternância de três semicondutores, IGBT, MOSFET e GTO. Os resultados de distorção harmônica de corrente na saída do conversor mostram um índice adequado para os três cenários, permitindo concluir que a mudança de semicondutores e a característica de carga não linear do conversor não piora o indicador.
\end{abstract}

\begin{abstract}
In this work two situations were proposed: i) the main one corresponds to validate through simulation in the software MATLAB / Simulink the use of a simple inverter whose nominal power of test is fractionated when compared with the high test power of the Back-to-Back converter (B2B), found in the technical literature in [1-3]. The converter is a key part of the TUT loading (TUT - Transformer Under Test). The apparent power at the output of the inverter was measured, as well as the active and reactive power from the network connected to the system. The result of the apparent power value measured in the converter is reduced compared to the power of the TUT, concluding that it is possible to perform the same test by replacing a $75 \mathrm{kVA}$ to a $5 \mathrm{kVA}$ converter. The other question concerns: ii) analyzing in the aspect of power quality about the harmonic distortion indicator, measured at the output of the power converter. For this work stage, the tests were performed in three moments, with alternation of three semiconductors, IGBT, MOSFET and GTO. The current harmonic distortion results at the output of the converter show an adequate index for the three scenarios, allowing to conclude that the semiconductor change and the nonlinear load characteristic of the converter does not worsen the indicator.
\end{abstract}

Keywords (Palavras chaves): Transformer, Test, Nominal, Inverter, Regenerative, Semiconductor.

\section{Introdução}

Este artigo apresenta uma análise baseada na teoria e simulação computacional de um sistema para ensaiar o carregamento de transformadores de potência emulando a operação regenerativa de uma carga com recirculação de energia. Alguns trabalhos [1-3] se diferenciam da topologia proposta neste trabalho e em relação a referência [4] ao utilizar um conversor atuando como uma fonte de tensão alternada, conectada em série com o transformador. Os testes em equipamentos submetidos a condições nominais se justifica no sentido de permitir prever condições reais de operação do sistema, bem como atuar em situações adversas de funcionamento do equipamento antes de sua real aplicação. Ao assegurar a confiabilidade do 
instrumento reforça-se a qualidade do processamento da energia no sistema ao qual o transformador está inserido. A possibilidade de ensaiar o sistema recirculando a energia envolvida do processo cumpre requisitos de eficiência energética, pois a potência consumida será de pequena ordem, neste caso para alimentar perdas no processo. Em uma segunda abordagem com o conversor de potência, são considerados e analisados separadamente os semicondutores IGBT, MOSFET e GTO na questão de impacto da qualidade da energia. A característica de não linearidade de cargas eletrônicas, em particular deste artigo sobre conversores, acrescenta-se um conteúdo harmônico na frequência fundamental de tensão e corrente elétrica do sistema [5], causando uma distorção harmônica e podendo violar níveis estabelecidos em norma regulamentadora [21]. Os trabalhos adotados como referência técnica neste artigo apresentam de diferentes maneiras o assunto de interesse deste trabalho e resumidamente serão descritos nesta seção. Nos trabalhos que correspondem às referências técnicas apresentadas em [1-3] encontra-se uma estrutura laboratorial que permite realizar testes de desempenho em transformadores de potência em condições fieis à realidade, o que se torna um desafio quando se exige um elevado nível de energia nos ensaios. O sistema emprega dois conversores com conexão back-to-back (B2B) de potência plena (mesma potência do transformador sob teste, "TUT" observado em [1], permitindo a regeneração de energia). Santos e colaboradores [5] apresentam um estudo da qualidade da energia elétrica acerca do impacto de tensões não senoidais ocasionadas por gerações harmônicas através de conversores eletrônicos de potência encontrados em sistemas de geração fotovoltaica. Em relação a topologias conversoras de potência, existem outras estruturas que realizam a simulação de carga para testes em equipamentos de diversas naturezas e são apresentadas nas referências [6-13], adotando o conversor B2B. Hogan e colaboradores [14] apresentam uma estrutura para estudos de uma microrrede, que não só representa o comportamento de cargas como também de fontes conectadas ao sistema. Há varias técnicas de detecção e controle de correntes e/ou potência [15-18] sendo exaustivamente estudadas objetivando a confiabilidade da operação da carga regenerativa. Mesmo em operação adequada realizada pelos autores da referência [1], é necessário o uso de um conversor de potência plena, ou seja, a potência do B2B deve no mínimo ser igualada a potência do transformador sob teste. Este trabalho propõe outra configuração, resultando em uma potência de conversor fracionada se comparada a potência do TUT. Posteriormente busca-se comparar o efeito que o conversor de energia causa na qualidade da energia, considerando três cenários de simulação com os componentes semicondutores IGBT, MOSFET e GTO.

\section{Configuração Proposta Características do Sistema}

A topologia adotada no esquema proposto é baseada no funcionamento de um transformador "phase shiff" utilizado em sistema de potência, que permite controlar o fluxo de potência ativa $(P)$ e reativa $(Q)$ entre as barras. Para atender este objetivo foram considerados os resultados de uma única simulação, usando o dispositivo IGBT no inversor de potência. A topologia da figura (3) facilita a compreensão da simulação na qual ilustra o esquema simplificado do circuito que foi adotado em todos os testes, exceto a particularidade do dispositivo semicondutor do inversor diferenciado em cada ensaio. Na figura 1, a barra 1 (B1) representa a tensão do enrolamento primário do transformador geral, que corresponde a $V_{1}^{\prime}$ da Eq. (1) e (2), a barra 2 (B2) representa a tensão do enrolamento secundário do transformador TUT, ou seja, $V_{2}$ da Eq. (1) e (2). A tensão $V_{E X T}$ é o inversor que atua como fonte de tensão e $X_{\text {TRAFOS }}$ representa toda a reatância e resistência do sistema elétrico, obtida dos transformadores mencionados. Por fim, delta $(\delta)$ corresponde à diferença de ângulo de tensão entre B1 e B2 da equação. Conhecidos os parâmetros da figura e aplicados na Eq. (1) e (2), consegue-se controlar a potência que circula nos transformadores com ajuste da tensão externa $\left(V_{E X T}\right)$ do inversor. Na configuração do sistema elétrico de potência deste artigo o inversor é conectado em série com os transformadores. 


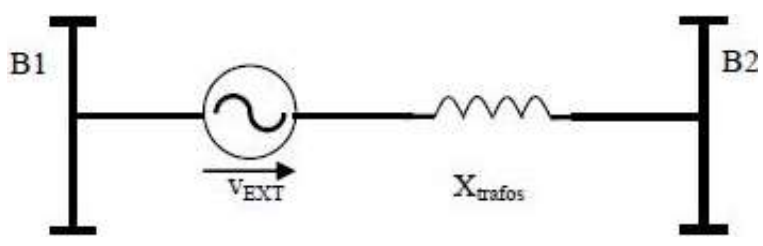

Figura 1: Esquema simplificado da topologia proposta.

$$
\begin{gathered}
P_{12}=\frac{V_{1}^{\prime} V_{2}}{X_{\text {trafos }}} \operatorname{sen}(\delta) \\
Q_{12}=\frac{\left(V_{1}^{\prime}\right)^{2}-V_{1}^{\prime} V_{2}}{X_{\text {trafos }}} \cos (\delta)
\end{gathered}
$$

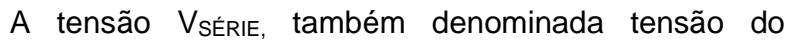
inversor na figura 2, está ligada no ramo de alta tensão e ocorre quando o barramento de baixa tensão (BT) representado pela tensão da rede $\left(\mathrm{V}_{\mathrm{REDE}}\right)$ está disponível. A análise estudada considera além das figuras anteriores a figura 3, cuja topologia apresentada foi simulada na plataforma MATLAB/Simulink. A tensão $\left(V_{R E D E}\right)$ é trifásica e fornece a tensão eficaz de linha de $220 \mathrm{~V}$ na frequência de $60 \mathrm{~Hz}$. Os transformadores do circuito representam transformadores de potência reais de distribuição de energia. Na alta tensão (AT) do sistema foi aplicada a tensão de $13,8 \mathrm{kV}$, coerente com o nível de tensão de uma rede elétrica de distribuição. A fonte $\left(V_{E X T}\right)$ é conectada em série no sistema através de três transformadores isoladores monofásicos idênticos. Estes transformadores fazem a adequação da tensão de saída da fonte com a tensão necessária na conexão série do sistema, o que permite impor a corrente de referência. Estima-se o valor inicial da tensão a ser introduzida em série com o sistema através da Lei de Kirchhoff das Tensões (LKT) ao circuito, que pode ser representado pela figura 2 considerando a configuração da figura 3.

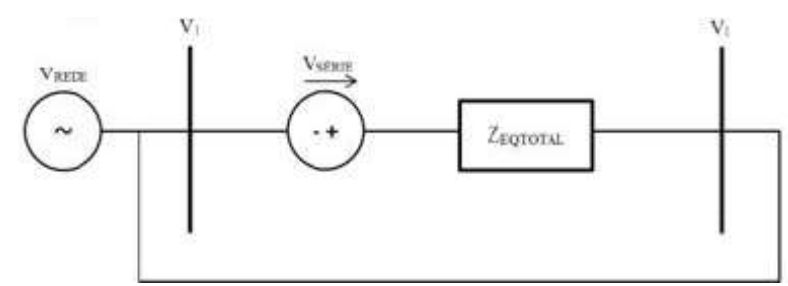

Figura 2: Malha do circuito equivalente para análise da tensão por LKT.

Eq. (3) equaciona as tensões da malha e se considera como impedância total do sistema ( $Z_{\text {EQTOTAL }}$ a resistência e a reatância dos transformadores. Como a tensão nas duas extremidades da malha são idênticas, obtém-se a tensão necessária introduzir para fazer circular a corrente nominal $\left(I_{N O M}\right)$, dada em (4):

$$
\begin{gathered}
\dot{V}_{1}+V_{\text {Série }}+I_{\text {NOM }} \times Z_{\text {EQTOTAL }}=\dot{V}_{1} \\
V_{\text {Série }}=I_{N O M} \times Z_{\text {EQTOTAL }}
\end{gathered}
$$

A corrente nominal $\left(I_{N O M}\right)$ corresponde ao valor de pico de 4,47 A referente ao transformador de $75 \mathrm{kVA}$ e de tensão de $13,8 \mathrm{kV}$. A impedância equivalente total do circuito corresponde a $178,36 \Omega$ e seu ângulo de fase vale $64,32^{\circ}$. Com base nos valores apresentados a tensão V SÉRIE calculada do circuito é igual a $792 \mathrm{~V}$. A tensão no barramento $\mathrm{cc}$ do inversor é igual a 400V, escolhida dentro da faixa de 300-600V dos transformadores monofásicos de conexão do inversor com o sistema. Além da faixa de tensão mencionada dos transformadores monofásicos, seus valores nominais de potência e frequência são respectivamente $1 \mathrm{kVA}$ e $60 \mathrm{~Hz}$. A frequência de chaveamento do inversor corresponde a aproximadamente $5 \mathrm{kHz}$.

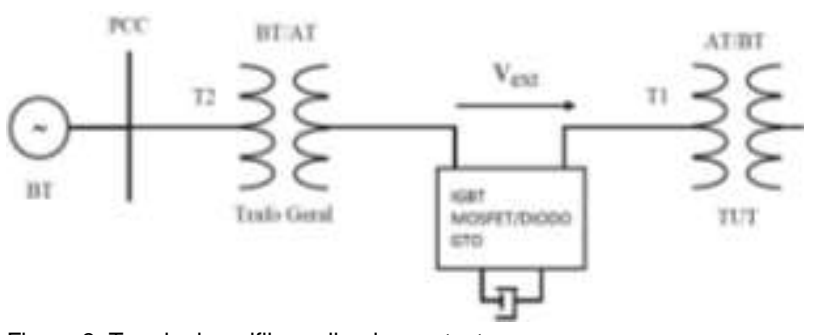

Figura 3: Topologia unifilar aplicada nos testes.

Para os testes com os semicondutores de potência quanto a sua influência na qualidade da energia, mais especificamente ao indicador de distorção harmônica de tensão e corrente (THD - Total Harmonic Distortion), foram realizados três ensaios. A única modificação de parâmetro ocorrida nos testes foi a alternância do semicondutor usado no inversor, ou seja, IGBT configurado inicialmente no inversor, MOSFET posteriormente e GTO finalizando os ensaios. O THD é um parâmetro usado para verificar o impacto harmônico em um sistema dado pela relação do valor efetivo da componente harmônica inserida na rede e o sistema fundamental, neste caso, $60 \mathrm{~Hz}$ [5]. No cálculo da distorção harmônica total considera-se a Eq. (5): 


$$
T H D=\frac{\sqrt{\sum_{n=2}^{\infty} I_{n}^{2}}}{I_{1}}
$$

A fórmula do $T H D$ mostra a soma das componentes de corrente harmônica do sistema e sua relação com a corrente fundamental do sistema (I1), a componente fundamental.

\section{Resultados}

A figura 4 corresponde a corrente nominal ( $\left.I_{N O M}\right)$ medida na alta tensão do sistema proposto para os ensaios com os semicondutores IGBT, MOSFET e GTO. Em ambos os ensaios, obteve-se o valor de corrente de 4,44 A que é o mesmo valor calculado para a corrente nominal do sistema. A alternância dos pares semicondutores não distorce a forma de onda medida, consequentemente não interfere no aspecto da qualidade da energia, já observada no shape da corrente.

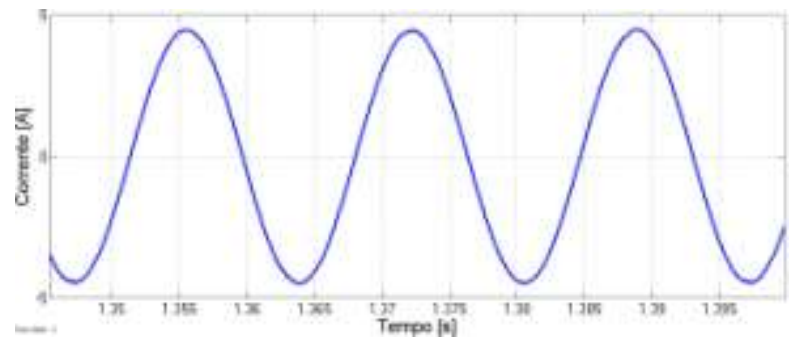

Figura 4: Corrente elétrica das três simulações dos semicondutores IGBT, MOSFET e GTO, que circula na alta tensão para $\mathrm{fp}=1$. Horizontal: $5 \mathrm{~ms} / \mathrm{div}$; vertical: $5 \mathrm{~A} / \mathrm{div}$.

A figura 5 apresenta 0 valor em percentual da magnitude da fundamental da corrente da figura anterior para os harmônicos de $1^{\mathrm{a}}$ a $16^{\underline{a}}$ ordem $(60 \mathrm{~Hz}$ - $1000 \mathrm{~Hz}$ ), e revela a taxa de distorção harmônica total $(T H D)$ de corrente proveniente do inversor. $O$ gráfico foi obtido para os três tipos de semicondutores para a análise do impacto de qualidade da energia oriunda do inversor, de natureza de carga não linear. 0 resultado de distorção harmônica de corrente de cada componente harmônica foi praticamente idêntico nos três cenários de testes, com quase a totalidade de medições registrando valores abaixo de $0.15 \%$ de magnitude harmônica de corrente. Com o somatório desses valores, o indicador de distorção harmônica total $(T H D)$ de corrente registrou o valor de $0,59 \%$ de conteúdo harmônico, bem abaixo dos valores limites definidos no PRODIST [21] para a qualidade da energia.

As figuras 6 e 7 apresentam os resultados mais importantes do trabalho, referente ao ensaio para a validação da topologia proposta neste artigo, objetivando viabilizar o ensaio de carregamento do transformador de potência sob teste (TUT) com um valor de potência do inversor bastante inferior do que visto em [1]. A figura 6 é obtida da medição de potência aparente na saída do inversor para comprovar a redução da potência ensaiada no mesmo. Dela, mostra-se a principal vantagem da proposta aqui apresentada para ensaio de transformadores sob carga: enquanto a bancada em [1], para ensaiar o transformador de $75 \mathrm{kVA}$, requer um conversor B2B dos mesmos 75 kVA, a bancada aqui descrita vai requerer um conversor simples de 5 kVA. A figura 7 apresenta o resultado de potência ativa $(P)$ e potência reativa $(Q)$ que a rede injeta na bancada proposta. $A$ figura mostra valores desprezáveis aplicados no circuito da bancada e confirma que o consumo de potência da rede é mínimo, demonstrando a característica regenerativa de energia do sistema.

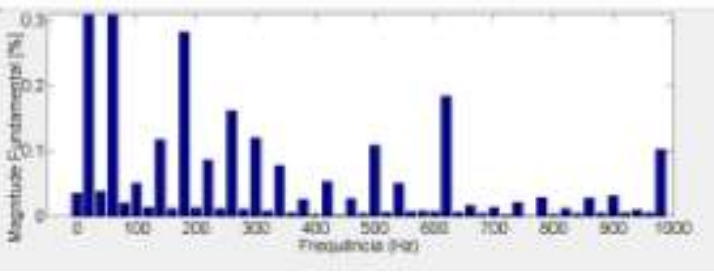

Figura 5: Distorção harmônica de corrente fornecida pelo inversor para $\mathrm{fp}=1$ para os três semicondutores, IGBT, MOSFET e GTO. Horizontal: $100 \mathrm{~Hz} /$ div; vertical: $0,1 \% /$ div.

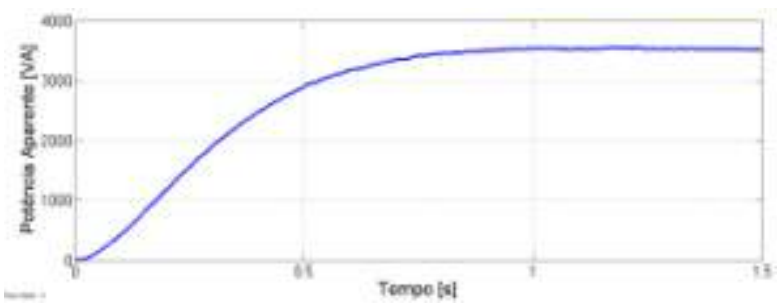

Figura 6: Potência aparente $S$ fornecida pelo inversor, 1000 VA/div Horizontal: $500 \mathrm{~ms} /$ div. 


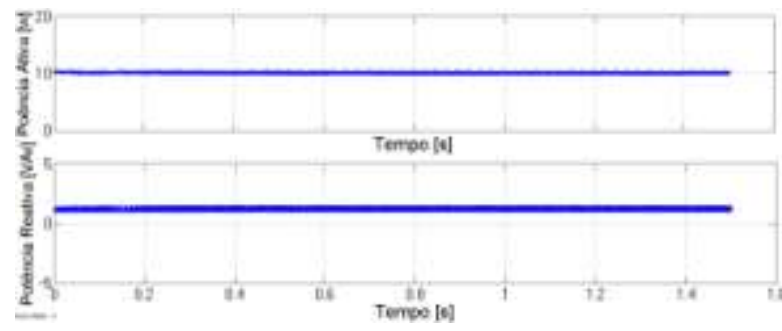

Figura 7: Potência ativa (superior, $10 \mathrm{~W} /$ div) e reativa (inferior, 5 VAr/div) fornecida pela rede à bancada (excluída a potência do inversor).

\section{Conclusões}

Este artigo apresentou a análise e simulação de uma bancada para ensaios de transformadores com carga cuja principal realce é empregar um conversor cuja potência é apenas uma fração da potência nominal do transformador. Os resultados apresentados nas figuras 6 e 7 confirmam que é possível ensaiar o transformador com potência nominal e fator de potência qualquer fazendo uso de um inversor de baixa potência comparativamente à potência do TUT. Foi ratificado pelos valores de potência que fluem da rede para a bancada, que a bancada proposta é de consumo de potência mínimo, operando como carga regenerativa. Os resultados quanto à análise do impacto de qualidade da energia elétrica em uma bancada com o uso de um inversor com a alternância dos dispositivos semicondutores mostraram que o índice de distorção harmônica de corrente $(T H D)$ foi o mesmo nos três semicondutores, com valores satisfatórios do conteúdo harmônico. Além disso, a característica de carga não linear emulada pelo inversor não ocasiona um prejuízo na qualidade da energia, fato que pode não se confirmar no aumento da presença de conversores eletrônicos largamente presentes, por exemplo, em sistemas fotovoltaicos.

\section{Agradecimentos}

Os autores agradecem a CAPES pelo apoio financeiro.

\section{Referências}

[1] C. V. Souza, F. F. Matos, G. M. Rezende, S. R. Silva, V. F. Mendes "Regenerative Active Electronic Load for Testing Power Transformers Under Linear and Nonlinear Conditions", Journal of Control, Automation and Electrical Systems February, Volume 27, Issue 1, pp 105-117, 2015.

[2] C. V. Souza, F. F. Matos, V. F. Mendes, I. J. Lopes, S. R. Silva, S. I.Seleme "Regenerative PWM Source for Power Transformer Loading Tests", Industrial Technology (ICIT), 2010 IEEE International Conference, 2010.
[3] G. M. Rezende, C. V. de Sousa, F. F. Matos, V. F. Mendes, P. C.Cortizo, W. C. Boaventura, et al. (2012). "Simulador de Cargas Não-Lineares Regenerativo em Teste de Transformadores de Potência".Anais do XIX Congresso Brasileiro de Automática (pp. 1583-1589).

[4] T. M. Galvão, D. S. L. Simonetti "Proposta de Conversor de Potência Parcial para Ensaios Regenerativos de Transformadores em Carga: Análise e Simulação", Conferência Brasileira de Qualidade da Energia Elétrica, CBQEE, 2017.

[5] K. A. G. Santos, P. M. Duarte, P. F. Ribeiro, P. M. Silveira "The Impact of Non-Sinusoidal Voltages on the Harmonic Generation of Power Electronics Converters.", Harmonics and Quality of Power (ICHQP), 2016 17th International Conference on 16-19 Oct. 2016. [6] B. Han, B. Bae, N. Kwak "Load Simulator with Power RecoveryCapability Based on Voltage Source Converter-Inverter Set", PowerEngineering Society General Meeting, 2007. IEEE, 2007.

[7] M. H. Bierhoff, F. W. Fuchs "Semiconductor losses in voltage sourceand current source IGBT converters based on analytical derivation",Power Electronics Specialists Conference, PESC, 04(4), 2836-2842,2004. [8] F. Blaabjerg, E. Chiarantoni, A. Dell'Aquila, M. Liserre, S. Vegura,"Analysis of the grid side behavior of a LCL-filter based three-phaseactive rectifier". In 2003 IEEE international symposium on industrial electronics. ISIE 03 (Vol. 2, pp. 775-780), 2003.

[9] J. A. Heerdt, D. F. Coutinho, S. A. Mussa, M. L. Heldwein .(2014). Control strategy for current harmonic programmed AC active electronic power loads. IEEE Transactions on Industrial Electronics, 61(8), 3810 3822.

[10] J. Alcalá, V. Cárdenas, H. Miranda, J. PérezRamírez and S. Charre, "Athree-phase back-to-back converter for reactive power compensation,current harmonic filtering and active power compensation," 2013 IEEE Energy Conversion Congress and Exposition, Denver, CO, 2013, pp.2371-2377.

[11] R. L. Klein, A. F. Paiva, M. Mezaroba, "Regenerative AC electronic load with LCL filter", 2012 10th IEEE/IAS international conference on industry applications (INDUSCON), (pp. 1-7), 2012.

[12] L. F. Guimarães, L. G. Kawahara, R. C. Annunziato, R. Gules. (2013). "Design and implementation of an electronic load". In Power Electronics Conference (COBEP), 2013 Brazilian (pp. 1075-1081).

[13] C. Roncero-Clemente, M. I. Milanes-Montero, V. M. Minambres-Marcos, E. Romero-Cadaval. (2011). "Three-phase regenerative electronic load to test shunt power conditioners". 2011 7th internationalconferenceworkshop compatibility and power electronics (CPE) (pp.178-183).

[14] D. J. Hogan, F. Gonzalez-Espin, J. G. Hayes, R. Foley, G. Lightbody,M. G. Egan, "Load and source electronic emulation using resonant current control for testing in a microgrid laboratory". In 2014 IEEE $5^{\text {th }}$ international symposium on power electronics for distributed generation systems (PEDG), pp. 1-7, 2014. [15] F. P. Marafão, D. I. Brandão, F. A. S. Gonçalves, H. K. M. Paredes. (2013). "Decoupled reference generator for shunt active filters using the conservative power theory". Journal of Control, Automation and Electrical Systems, 24(4), 522-534.

[16] Rodriguez, P., Teodorescu, R., Candela, I., Timbus, A. V., \& Blaabjerg,F. (2006). "New positive- 
sequence voltage detector for gridsynchronization of power converters under faulty grid conditions". In 37th IEEE power electronics specialists conference (pp. 17).

[17] Teodorescu, R., Blaabjerg, F., Liserre, M., \& Loh, P. C. (2006)."Proportional-resonant controllers and filters for grid-connected voltagesource converters". IEE Proceedings-Electric Power Applications,153(5), 75062.

[18] Twining, E., \& Holmes, D. G. (2003). "Grid current regulation of a three-phase voltage source inverter with an LCL input filter". IEEE Transactions on Power Electronics, 18(3), 888-895.
[19] UNICAMP - Junções em Semicondutores - Cap. 4 - 2017. Disponível em

<http://www.ccs.unicamp.br/cursos/fee107/download/ca p04.pdf >. Acesso em: 09/08/2017.

[20]UNICAMP - Introdução aos semicondutores. Disponível

em

<http://www.dsif.fee.unicamp.br/ fabiano/EE530/PDF/T exto\%20\%20F\%EDsica\%20dos\%20Semicondutores.pd $\mathrm{f}>$. Acesso em 09/98/2017.

[21] ANEEL - Agência Nacional de Energia Elétrica PRODIST - Módulo 8 - "Qualidade de Energia Elétrica”, 4 ${ }^{\text {a }}$ Revisão, 2012. Disponível em $<w w w$.aneel.gov.br>.Acesso em: 09/08/2017. 